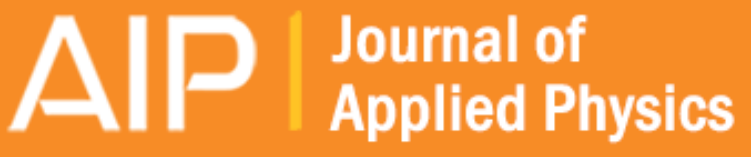

Influence of sample width on the magnetoresistance and planar Hall effect of $\mathrm{Co} / \mathrm{Cu}$ multilayers

S. C. Lima and M. N. Baibich

Citation: Journal of Applied Physics 119, 033902 (2016); doi: 10.1063/1.4939924

View online: http://dx.doi.org/10.1063/1.4939924

View Table of Contents: http://scitation.aip.org/content/aip/journal/jap/119/3?ver=pdfcov

Published by the AIP Publishing

\section{Articles you may be interested in}

Influence of interfacial scattering on giant magnetoresistance in $\mathrm{Co} / \mathrm{Cu}$ ultrathin multilayers

J. Appl. Phys. 109, 033910 (2011); 10.1063/1.3544471

High-field magnetoresistance, giant magnetoresistance, and superparamagnetism in Co/Cu multilayers

J. Appl. Phys. 91, 8590 (2002); 10.1063/1.1451890

Magnetization processes in $\mathrm{Co} / \mathrm{Cu}$ multilayers with low magnetoresistive hysteresis

J. Appl. Phys. 86, 1611 (1999); 10.1063/1.370935

Correlation of domain processes and magnetoresistance changes as a function of field and number of bilayers in Co/Cu multilayers

J. Appl. Phys. 81, 3775 (1997); 10.1063/1.365505

Observation of domain dynamics in giant magnetoresistive Co-Cu-based polycrystalline multilayers

J. Appl. Phys. 81, 4582 (1997); 10.1063/1.364749

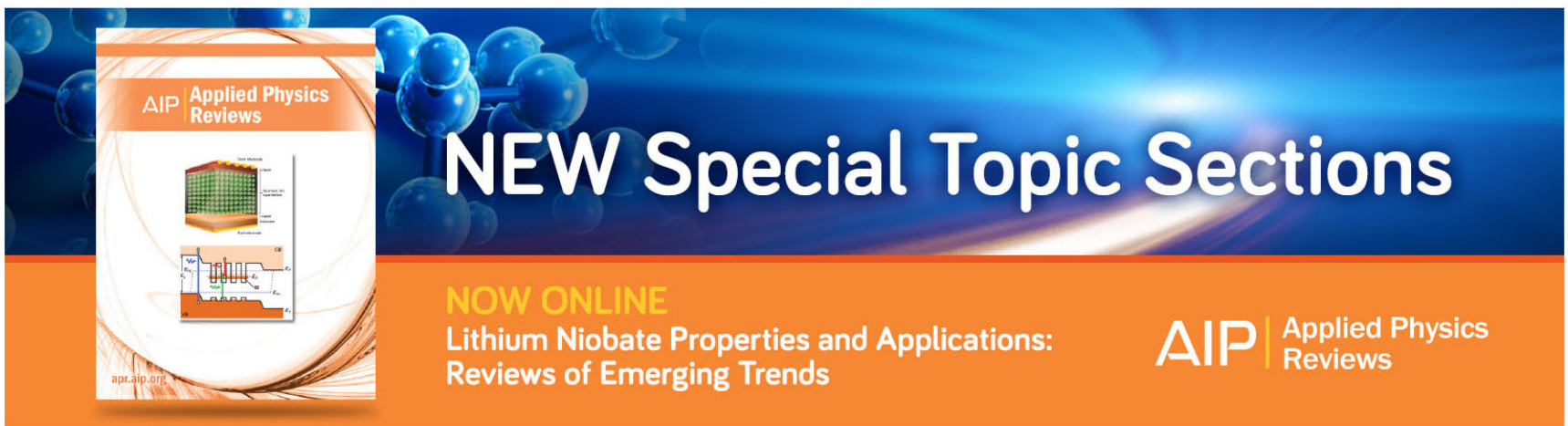




\title{
Influence of sample width on the magnetoresistance and planar Hall effect of $\mathrm{Co} / \mathrm{Cu}$ multilayers
}

\author{
S. C. Lima and M. N. Baibich \\ Instituto de Física, Universidade Federal do Rio Grande do Sul, Av. Bento Gonçalves 9500, CP 150151, \\ 91501-970 Porto Alegre, RS, Brazil
}

(Received 27 October 2015; accepted 4 January 2016; published online 19 January 2016)

\begin{abstract}
We have studied $\mathrm{Co} / \mathrm{Cu}$ multilayers to understand the effect of the sample's width on their magnetoresistance (MR). By keeping constant both the length and the thickness and exploring the widths within the range of usual magnetic domain sizes in those nanostructures, we were able to observe effects on the MR curve, as well as infer linked changes in the magnetization process. Associating MR and Planar Hall Effect (PHE) measurements, coupled to an analysis of the MR plots' symmetry, we were able to establish that, apart from the expected antiferromagnetic coupling, reducing the width forces the magnetization to stay aligned to the current channel, thus inducing more symmetric, closer to model Giant Magnetoresistance (GMR). Also, the sample edges might contribute by adding extra coupling through magnetostatic interaction. The added effects result in a counter-intuitive trend that goes from the near ideal wide samples through intermediate sizes with fairly abrupt changes in MR, and finally to the closer to bell-shaped ideal GMR at narrow widths. C 2016 AIP Publishing LLC. [http://dx.doi.org/10.1063/1.4939924]
\end{abstract}

\section{INTRODUCTION}

A few decades past the discovery and subsequent use of GMR, ${ }^{1,2}$ the role of the sample size in defining the mechanisms of the magnetization and transport is still not perfectly understood. In particular, the range of sizes not used for applications was seldom studied.

As can be seen in the specialized literature, most of the studies focus either on fairly wide (or very narrow) samples, leaving the range of the "transition widths" unexplored.

In wide samples, the main subjects are either the magnetic coupling of the layers (linked to the process of magnetization on that account $)^{3-6}$ or the description of the magnetotransport processes in Current in Plane (CIP) or Current Perpendicular to Plane (CPP) conduction. ${ }^{7,8}$

On the other hand, most authors that have dealt with very narrow spin- or pseudo spin-valve systems, probably stimulated by the perspective of applications, were mainly focused on the magnitude variation of the GMR, ${ }^{9-11}$ not paying much attention to the changes in its magnetic field response.

As a matter of fact, the GMR can be used to detect changes in the magnetic structure of the sample as an alternative to studying the evolution of the magnetization processes using more conventional methods such as magnetization (by SQUID, AGFM, or VSM) or magnetic force microscopy (MFM), for instance. ${ }^{12}$

Additionally, planar Hall effect (PHE) measurements are a useful tool to investigate the magnetization process, as it depends on the direction of the magnetization in each magnetic layer. This is information that cannot be obtained directly from GMR since this depends on the relative orientation between neighbouring magnetic layers only. ${ }^{13}$

We have chosen to use $\mathrm{Co} / \mathrm{Cu}$ multilayers for this investigation, for this system presents large GMR and has been extensively studied, providing a substantial knowledge background. Moreover, conditions for antiferromagnetic coupling between cobalt layers are well known for this type of structure. ${ }^{14}$ As pointed out earlier, the influence of the sample width in these coupling processes was not adequately explored.

Accordingly, we present this contribution to the understanding of the phenomena arising from the miniaturization of these magnetic structures, specifically in a range close to typical sizes for magnetic domains in these multilayers.

\section{EXPERIMENTAL}

A $\quad \mathrm{Si} / \mathrm{SiO}_{2}(\sim 1 \mu \mathrm{m}) / \mathrm{Ta}(50 \AA) /[\mathrm{Cu}(20 \AA) / \mathrm{Co}(14 \AA)]_{10} / \mathrm{Cu}$ (50 $\AA$ ) multilayer structure was deposited by DC magnetron sputtering (base pressure $<5 \times 10^{-8}$ Torr; working pressure around 3 mTorr). Deposition rates were $1.3,1.4$, and $1.8 \AA / \mathrm{s}$ for tantalum, copper, and cobalt, respectively, onto a clean thermally oxidized silicon wafer. The MR read about $6 \%$ at room temperature in wide $(\mathrm{w}>1 \mathrm{~mm}$ ) samples.

The chosen thickness of the copper layer corresponds to the second maximum of the AF coupling. This is in order to guarantee the absence of pinholes in the non-magnetic layers, but also providing sizeable GMR to use as a probe of the magnetization processes in our study of the role played by the width of the samples.

After preparation of the multilayers, the sample width was reduced using standard optical lithography with wet etching by diluted solutions of nitric and hydrofluoric acids on a laser writer defined diagram, as seen in Fig. 1.

In view of the range of sizes explored, the small width of the current channel forces the easy magnetic axis to be roughly on the same direction. On the samples studied, we have contacts for MR with $10 \mu \mathrm{m}$ between leads, as well as the needed connections for Hall effect.

A four-point method was used with AC $(27 \mathrm{~Hz})$ currents always under $10 \mu \mathrm{A}$ and a lock-in technique for detection. The data were registered in $\mathrm{R}$ - $\theta$ mode, and no significant change was seen in phase $\theta$ when the magnetic field is 




FIG. 1. Schematics of a patterned sample showing the electrical connections for MR and PHE using a Tabletop Micro Pattern Generator model $\mu$ PG 101 (Heidelberg Instruments Mikrotechnik $\mathrm{GmbH}$ ). The inset is a zoom into the active sample region; $w$ is the reported width of the sample.

altered, i.e., no magnetoimpedance effect (MI) was observed for this low frequency. Also, in keeping the current within this limit, we did not observe changes in the resistance as a function of time, indicating no warming/self-annealing effects.

\section{RESULTS AND DISCUSSION}

Figure 2 presents the normalized MR for different sample widths, with the magnetic field (always in the plane of the sample) applied either parallel or perpendicular to the direction of the sample's current channel. As we concentrate in discussing the changes in magnetic response, all plots are



FIG. 2. Normalized room temperature MR for fields applied either parallel or perpendicular to the easy axis; $w$ is the width of the sample. The plotted lines are guides for the eyes. normalized for easy comparison. Notice the difference in the shape of the curves, especially when the applied field is perpendicular to the easy axis.

The $30 \mu \mathrm{m}$ wide sample is similar to the non-patterned one, that is, the curves match the observed peaks at all fields. However, in reducing the width, we easily see changes in the MR field dependence. Also, for same width samples, the usual differences in switching field-axis orientations are observed, but this tends to be more pronounced for smaller widths.

\section{A. Coercive field dependence}

In AF-coupled $\mathrm{Co} / \mathrm{Cu}$ multilayers, the coercive field $\left(\mathrm{H}_{\mathrm{C}}\right)$ coincides with the highest resistance in the MR curve, ${ }^{15,16}$ since null magnetization implies best antiparallel orientation between neighbouring layers. Figure 3 shows the coercive field as a function of sample width for fields applied either parallel or perpendicular to the easy axis. Again, the differences seen for each orientation are more pronounced when the sample becomes narrower.

Also, we find that wider samples follow a quite linear trend between coercive field and $1 / \mathrm{w}$, similar to what is seen in other works associated to the influence of Hemagnetizing field. ${ }^{17,18}$ However, as the in-plane demagnetizing factor should be below $10^{-3}$, this does not seem to be a significant factor here.

Many effects might lead to increase $\mathrm{H}_{\mathrm{C}}$ in micrometers strips, for instance, a gradual tendency for samples to behave like a single domain on reducing width, ${ }^{19}$ and some pinning enhancement that should naturally be expected at the samples' irregular edges.

Also, an effective sample width reduction for electronic transport can be envisaged (by the appearance of a "dead" zone of about $200 \mathrm{~nm}$ according to our calculations), which could be a result of the not-so-perfect resist layer coverage, or non-homogeneous corrosion, among many other possible imperfections inherent to the optical lithography process.

All mentioned effects tend to increase the coercive field, but those do not seem to be the only factors, as the $2-\mu \mathrm{m}$ wide sample has its measured $\mathrm{H}_{\mathrm{C}}$ significantly smaller than

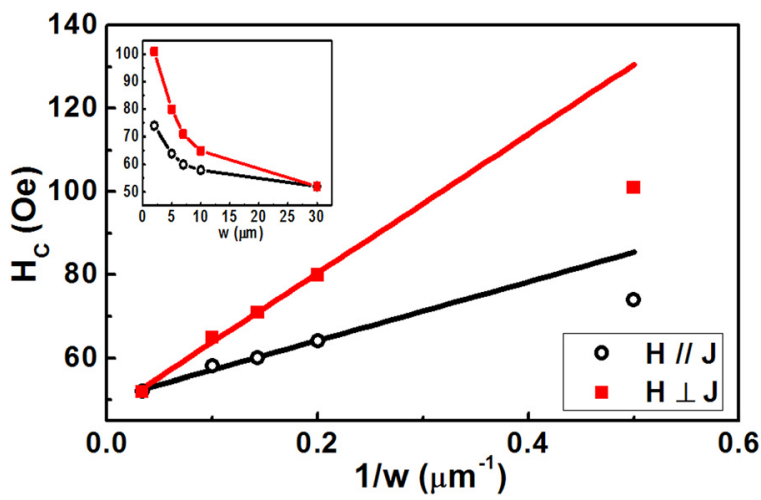

FIG. 3. The coercive field $\left(\mathrm{H}_{\mathrm{C}}\right)$ as a function of inverse sample width (the lines are linear regressions over the first four points). The inset shows a standard $\mathrm{H}_{\mathrm{C}}$ vs. width plot. 
the extrapolation from wide towards small widths for both the field applied perpendicular or parallel to the easy axis.

The surface area of the contact pads in our samples might be one of the culprits in that discrepancy: in wider structures, the nucleation of domains can occur as easily in the current channel as in the pads, making the latter to have negligible contribution. However, the role of the pads is vital in narrower structures. When this happens, the formation of domain walls at the intersections between pads and current channel is easier than in the inter-pads region, promoting the propagation of domain walls from this larger area into the structure, producing quite different results. ${ }^{18}$ Any sort of change in the coupling between layers might also contribute for this.

\section{B. MR profile dependence}

A most interesting aspect to explore in these samples is the MR plots' profile change with size. If one takes an actual measurement sweep as, for instance, scanning from the largest negative field to full positive, a clear asymmetry around the coercive field is seen for wider specimens, i.e., the resistance varies faster before reaching $\mathrm{H}_{\mathrm{C}}$ than it does after it, as seen in Fig. 4, where an ascending branch of the MR plot is shown with the side at the left of $\mathrm{H}_{\mathrm{C}}$ reflected to the right in order to show the difference between the two sides about the maximum resistance. On reducing the sample width, a trend to symmetrisation is observed, i.e., the resistance as a function of field tends to be similar on both sides of the coercive field. This is seen by the decreasing gap between the up and down resistance branches, which is clearer for the narrowest sample with fields applied perpendicular to the easy axis: the profile is closer to a bell-shaped curve (centered on the coercive field) and suggests that the layers are better-coupled. ${ }^{20}$

This, according to semi-classical descriptions of GMR, is likely connected to a change in the magnetization reversal mechanism on reducing the width.

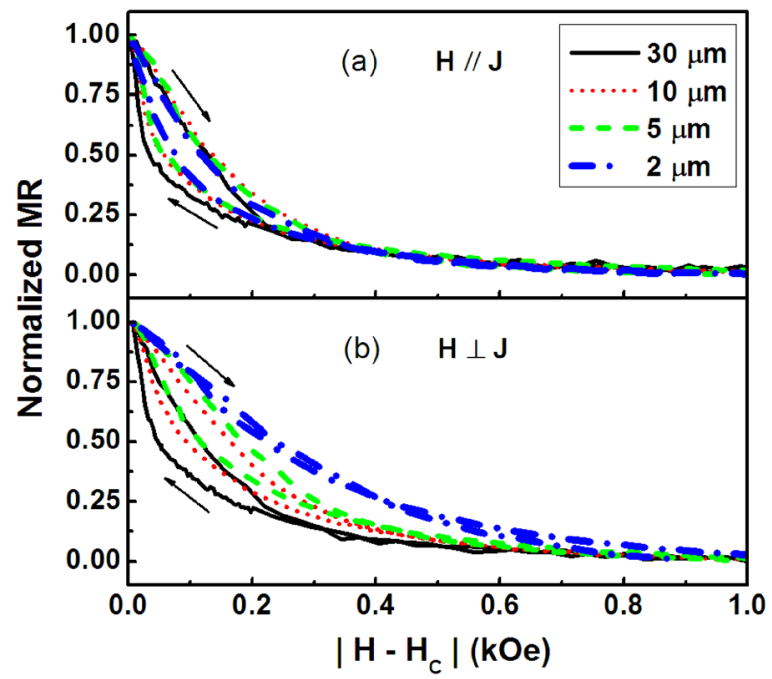

FIG. 4. Comparison of the upward MR branches reflected about the coercive field: (a) parallel and (b) perpendicular magnetic fields with respect to the current flow direction.
The PHE can help clarify these results, as it originates from the same anisotropy source that brings anisotropic MR (AMR), and is seen whenever the resistivity depends on the angle $\theta$ between the magnetization $M$ and current density $J$.

Both effects are proportional to the square of the total magnetization. However, while AMR has $\cos ^{2} \theta$ dependence, PHE depends on $\sin 2 \theta$. From the functional form of both effects, one sees that the angle derivative of the AMR results in a plot similar to PHE.

In Figure 5, we show both the PHE and an ascending branch of $d M R / d H$ for a 5- $\mu \mathrm{m}$ wide sample at different relative field/current orientations.

As seen in Fig. 5(a), for $\mathrm{H} / / \mathrm{J}$, i.e., when $\alpha$ (the angle between the applied magnetic field and direction of current flow) is zero, the PHE voltage remains near zero from the saturation field to point "A." This is compatible with a process in which the magnetization vectors of neighbouring layers (or magnetic domains in neighbouring layers) rotate in opposite directions, with equivalent angular change relative to the field direction, in a scissors-like movement (yielding a rather standard coupled-multilayer magnetization evolution), which results in total magnetization reduction, but not changing the resulting direction (as expected for the samples have an even number of Co layers, and all have the same nominal thickness), satisfying a null PHE condition.

After point "A," the signal suddenly grows (peak at point "B") and then returns to "C." From that point on, it tends to return to the saturation value, joining the other

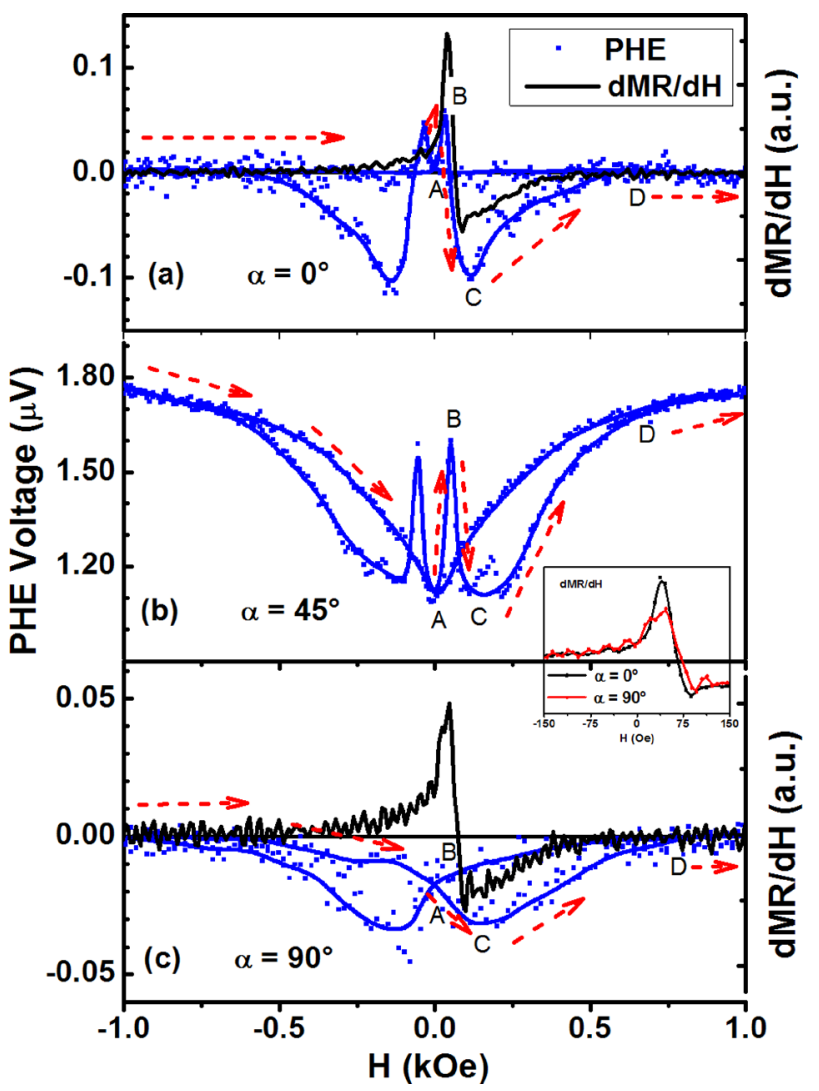

FIG. 5. PHE for a $5 \mu \mathrm{m}$ wide sample with different angles between applied magnetic field and current flow $\left(\alpha=0^{\circ}, 45^{\circ}\right.$ or $\left.90^{\circ}\right)$. The obtained $d M R / d H$ is also plotted to show the correlation between both quantities. The blue lines are guides for the eyes. 
branch at "D," completing the PHE curve. This means that, for field values between " $\mathrm{A}$ " and " $\mathrm{D}$," the net magnetization does not follow the same direction of the applied field (or current direction), i.e., the "scissors movement" no longer works.

At $\alpha=45^{\circ}$ (Fig. 5(b)), when the sample is saturated, the magnetic layers are aligned to the applied field and show maximum PHE. Then, decreasing the field, the scissors movement starts, reducing the total magnetization without change in direction (as in $\mathrm{H} / / \mathrm{J}$ ) and, consequently, a monotonic decrease of PHE up to point " $\mathrm{A}$ " is seen.

After point "A," the curve shows features inherent to the broken scissors-movement at the same field range as for $\alpha=0^{\circ}$, i.e., the appearance of an abrupt peak between the " $\mathrm{A}$ " and " $\mathrm{B}$ " points, plus another broad peak including the "C" and "D" points.

By comparing both plots, we infer that the differences are almost exclusively due to the difference between the angles $M \angle J$ used for the measurement, but the magnetic evolution processes are similar in both cases, which might explain the similarity between the MR curves obtained for $\alpha=0^{\circ}$ and $45^{\circ}$ (not shown).

Associated to this imbalance between magnetization directions for different layers, responsible for the PHE signal (mainly the sharp peak), one expects a corresponding change in $d M R / d H$. This might come either from the GMR fraction (from a fast change in relative alignment between adjacent layer magnetizations) or from AMR (due to an angle change with respect to the current flow, as seen by $d A M R / d \theta$ ). However, the relative fractions of each of the GMR and AMR contributions are not known.

The $d M R / d H$ curve shows two peaks (one positive and one negative) whose difference is directly associated to the asymmetry of the MR curve and is linked to the PHE plot, especially the sharp peak seen at the "A" to "B" region.

For $H \perp J\left(\alpha=90^{\circ}\right.$; Fig. 5(c)), a null PHE condition is again reached at saturation field, and a rather small PHE for all field values with no abrupt changes, as previously mentioned for the "A" to "B" range. As a result, no abrupt changes in the MR slope are seen (inset in Fig. 5: the $d M R /$ $d H$ plot for $\alpha=90^{\circ}$ shows a smaller peak than that for $\alpha=0^{\circ}$ ). In this case, a simpler evolution of the magnetization might be present, with no sudden variation of the magnetic state. The PHE behaviour from " $C$ " to " $D$ " suggests a small unbalance between the direction and/or magnitude of the magnetizations of alternate layers but is weak compared to other orientations.

Summing it up, the (anti) symmetry of $d M R / d H$ with respect to $\mathrm{H}_{\mathrm{C}}$ reflects the above-mentioned change in profile. Notice that measurements with the field parallel to the sample's easy axis have larger and sharper peaks in the $d M R / d H$ plot and, associated to it, a peak in PHE at the same applied field, which disappears when $H \perp J$. This also results in both a smoother derivative for this orientation and a monotonic PHE associated to lower amplitude.

To explain what is seen in our results, there must be a change in magnetization reversal scheme. These results should be related to those by Aitchison et al., ${ }^{21}$ who observed a sudden change in the direction of magnetization seen by magnetic Lorentz microscopy in certain regions of samples with large asymmetric-MR curves, while no such abrupt magnetization changes were seen for symmetric-MR ones. The asymmetry was attributed to the presence of a different magnetic domain distribution in each layer of the stack, i.e., there would not be good correlation between corresponding domains in neighbouring cobalt layers.

From the above-mentioned reasoning, the more symmetrical MR curve should refer to the situation where the magnetization in each domain of a layer is correlated to its neighbouring layers, evolving by coherent rotation between magnetizations and without noticeable individual depinning of specific domains. This correlation could be improved by increasing the $\mathrm{AF}$ coupling energy.

In fact, the rise of the branch crossings at $\mathrm{H}=0$ in the MR plots (see Fig. 2) can be seen as an indication that the magnetic layers are better coupled/correlated. ${ }^{4}$

A well coupled condition could be achieved, for instance, by intensifying the RKKY coupling, as usually seen in samples with $\mathrm{Cu}$ layer thicknesses at the first (and most intense) peak $(t \sim 10 \AA)$, which reveal bell-shaped curves around $\mathrm{H}=0$. Besides, increasing the number of bilayers (up to about 20-30) can raise the antiferromagnetically coupled fraction of the total sample area in $\mathrm{Co} / \mathrm{Cu}$ multilayers. ${ }^{22}$

However, our results show that the coupling is also affected by the reducing sample width sizes. This effect seems to be related to similar sizes for sample width and usual magnetic domain size at the magnetic remnant state (typically $0.5-1.5 \mu \mathrm{m}$ for wider $\mathrm{Co} / \mathrm{Cu}$ films ${ }^{16,23}$ ).

When the sample width is reduced, the growth and orientation of the magnetic domains are limited by the edges. This implies in changes in the magnetic evolution by the approach of a monodomain state, therefore enhancing interlayer correlation.

Another important aspect to be considered is the relative increase of the magnetostatic contribution. The surface magnetic charges associated to magnetic domains close to the sample edges in neighbouring layers do interact with each other. The condition that minimizes the energy of this interaction tends to promote AF coupling between these more external domains. In narrow samples, as the fraction of domains near the edges is forcibly larger, it leads to an enhanced overall $\mathrm{AF}$ coupling that, in turn, brings the observed effects on MR and PHE.

\section{CONCLUSIONS}

We produced DC magnetron-sputtered $\mathrm{Co} / \mathrm{Cu}$ multilayers and subsequently reduced their lateral size by optical lithography. We have shown that the MR features are affected by the width of the samples. The contact pads had influence on the results only when its dimensions were comparable to the sample width.

Also, the shape of the MR plots is related to the magnetic evolution of the $\mathrm{Co} / \mathrm{Cu}$ multilayers. Better symmetry around the coercive field is associated to coherent rotations between adjacent magnetic layers, which indicate an increase of AF interlayer coupling. Moreover, asymmetric curves were associated to weaker coupling, thus allowing 
more independent magnetic evolution for each layer, resulting in non-monotonic MR changes.

By shrinking the sample width, the adjacent cobalt layers are forced to stay better correlated to each other, suppressing possible misalignments between (total) magnetization and external field. This correlation may arise not only from limiting the freedom of domain directions by reducing the sample width but also due to the relevance of magnetostatic interactions at the edges. Unfortunately, which of these factors is dominant is not known.

\section{ACKNOWLEDGMENTS}

We thank the Laboratório de Conformação Nanométrica for the samples' growth and Laboratório de Microelectrônica for the complete lithographic process, both at IF-UFRGS. This work has been partially supported by CNPq (Brazil).

${ }^{1}$ M. N. Baibich, J. M. Broto, A. Fert, F. N. Van Dau, F. Petroff, P. Etienne, G. Creuzet, A. Friederich, and J. Chazelas, Phys. Rev. Lett. 61, 2472 (1988).

${ }^{2}$ J. M. Daughton, J. Magn. Magn. Mater. 192, 334 (1999).

${ }^{3}$ C. Christides, S. Stavroyiannis, N. Boukos, A. Travlos, and D. Niarchos, J. Appl. Phys. 83, 3724 (1998).

${ }^{4}$ G. J. B. Rodriguez, L. G. Pereira, M. G. M. Miranda, A. B. Antunes, and M. N. Baibich, J. Magn. Magn. Mater. 214, 78 (2000).

${ }^{5}$ J. N. Chapman, J. Rose, P. R. Aitchison, H. Holloway, and D. J. Kubinski, J. Appl. Phys. 86, 1611 (1999).
${ }^{6}$ P. R. Aitchison, J. N. Chapman, H. Holloway, D. J. Kubinski, and M. Parsons, J. Appl. Phys. 87, 5753 (2000).

${ }^{7}$ S. Zhang and P. M. Levy, J. Appl. Phys. 69, 4786 (1991).

${ }^{8}$ K. M. Schep, P. J. Kelly, and G. E. W. Bauer, Phys. Rev. Lett. 74, 586 (1995).

${ }^{9}$ S. C. Sanders, R. W. Cross, S. E. Russek, A. Roshko, and J. O. Oti, J. Appl. Phys. 79, 6240 (1996).

${ }^{10}$ F. J. Castaño, S. Haratani, Y. Hao, C. A. Ross, and H. I. Smith, Appl. Phys. Lett. 81, 2809 (2002).

${ }^{11}$ D. Morecroft, B. B. Van Aken, J. L. Prieto, D.-J. Kang, G. Burnell, and M. G. Blamire, J. Appl. Phys. 97, 054302 (2005).

${ }^{12}$ T. Ono, H. Miyajima, K. Shigeto, and T. Shinjo, Appl. Phys. Lett. 72, 1116 (1998)

${ }^{13}$ Z. Q. Lu, G. Pan, J. Li, and W. Y. Lai, J. Appl. Phys. 89, 7215 (2001).

${ }^{14}$ D. H. Mosca, F. Petroff, A. Fert, P. A. Schroeder, W. P. Pratt, Jr., and R. Loloee, J. Magn. Magn. Mater. 94, L1 (1991).

${ }^{15}$ Y. Ueda, T. Houga, H. Zaman, and A. Yamada, J. Solid State Chem. 147, 274 (1999).

${ }^{16}$ J. A. Borchers, J. A. Dura, J. Unguris, D. Tulchinsky, M. H. Kelley, C. F. Majkrzak, S. Y. Hsu, R. Loloee, W. P. Pratt, Jr., and J. Bass, Phys. Rev. Lett. 82, 2796 (1999).

${ }^{17}$ E. Y. Chen, S. Tehrani, T. Zhu, M. Durlam, and H. Goronkin, J. Appl. Phys. 81, 3992 (1997).

${ }^{18}$ K. Shigeto, T. Okuno, T. Shinjo, Y. Suzuki, and T. Ono, J. Appl. Phys. 88, $6636(2000)$

${ }^{19}$ J. H. J. Fluitman, Thin Solid Films 16, 269 (1973).

${ }^{20}$ C. H. Marrows, M. Perez, and B. J. Hickey, J. Phys.: Condens. Matter. 18, 243 (2006).

${ }^{21}$ P. R. Aitchison, J. N. Chapman, D. B. Jardine, and J. E. Evetts, J. Appl. Phys. 81, 3775 (1997).

${ }^{22}$ H. A. M. van den Berg and G. Rupp, IEEE Trans. Magn. 30, 809 (1994).

${ }^{23}$ S. Langridge, J. Schmalian, C. H. Marrows, D. T. Dekadjevi, and B. J. Hickey, J. Appl. Phys. 87, 5750 (2000). 